\title{
Chemical pancreatectomy: an unconventional approach to preventing autodigestion in pancreatitis
}

\author{
Rodger A. Liddle \\ Department of Medicine, Duke University Medical Center, and Department of Veterans Affairs Health Care System, Durham, North Carolina, USA.
}

\begin{abstract}
Due to progressive inflammation, chronic pancreatitis destroys both the exocrine and endocrine pancreas and sensitizes pancreatic nerves, leading to unremitting pain. Unfortunately, there are no treatments for pancreatic inflammation and approaches to ameliorate pain are suboptimal. Pancreatic inflammation is particularly problematic because damage to acinar cells causes local release of digestive enzymes, which initiate pancreatic autodigestion. The combination of autodigestion and inflammation is unique to pancreatitis and undoubtedly contributes to the difficulty in devising effective treatments. In this issue of the $J C I$, Saleh et al. describe a nonsurgical technique to ablate pancreatic acinar cells, thus eliminating the source of digestive enzymes and preventing autodigestion. In mice and a nonhuman primate model, this approach effectively reduced inflammation and pain while preserving islet cell function. These findings support the concept that ongoing acinar cell damage is at the root of chronic pancreatitis and provide a possible strategy for clinical treatment.
\end{abstract}

\section{Chronic pancreatitis}

Picture yourself in a hospital room where your son has been admitted with pancreatitis. This is his third attack, and he is only 11 years old. You should know what to expect since his father and uncle suffer bouts of excruciating abdominal pain and diabetes from an inherited form of chronic pancreatitis. A doctor enters and says, "We are going to inject acetic acid into his pancreatic duct." Instinctively, you reply, "You are going to do what?"

Chronic pancreatitis is a debilitating disease characterized pathologically by inflammation, atrophy, and fibrosis of the pancreas and clinically by recurrent abdominal pain, pancreatic enzyme insufficiency, and often, diabetes mellitus (1). Chronic pancreatitis is most commonly associated with excessive alcohol use, although genetic causes, such as muta- tions in cationic trypsinogen PRSS1 (2), the (trypsin inhibitor gene SPINK1 (3), chymotrypsinogen C CTRC $(4,5)$, and the cystic fibrosis gene $\operatorname{CFTR}(6,7)$, along with other polymorphisms, underlie hereditary or "idiopathic" pancreatitis. Hereditary pancreatitis, as exemplified in the fictional case above, is notorious for its childhood onset and for its subjects' increased lifetime risk of pancreatic cancer (8). The pathophysiology of pancreatitis involves two fundamental processes - autodigestion and inflammation - that in combination render the disease difficult to treat. Autodigestion occurs through the premature activation of pancreatic enzymes within the gland itself. Acinar cell necrosis is followed by an inflammatory response that, if unchecked, amplifies tissue damage. Recurrent attacks cause chronic pancreatitis. Longstanding, progressive

\section{Related Article: https://doi.org/10.1172/JCl143301}

Conflict of interest: The author receives royalty payments from UpToDate and is listed as an inventor on a pending patent application through Duke University (PCT/US2020/053024).

Copyright: (c) 2021, American Society for Clinical Investigation.

Reference information: / Clin Invest. 2021;131(3):e146210. https://doi.org/10.1172/JCl146210.

chronic pancreatitis leads to pancreatic atrophy with loss of exocrine and endocrine function. Although the pancreas has considerable exocrine reserve, malabsorption occurs when more than $90 \%$ of the gland has been destroyed (9).

The worst symptom for most patients with chronic pancreatitis is abdominal pain. Some patients experience repeated episodes of pain that resemble attacks of acute pancreatitis, but they may also have extensive pain-free periods. In other patients, the pain is constant, but may wax and wane in intensity. Unfortunately, there are no specific therapies for pancreatitis and the mainstays of chronic pancreatitis pain treatment are opioid and nonopioid analgesics, celiac plexus block, and surgery. These approaches do nothing to prevent the progression of inflammation that may ultimately destroy acinar cells and pancreatic islets. As a result, digestive enzyme deficiency and diabetes mellitus develop.

Surgery for chronic pancreatitis is performed to relieve obstructions of the pancreatic duct that contribute to pain or recurrent episodes of pancreatitis or to treat other complications, such as pseudocysts, necrosis, or duct disruption. Surgical pancreatectomy, usually with islet autotransplantation (TPIAT), has been employed as a last resort for patients with unrelenting pain or patients at high risk for developing pancreatic cancer (e.g., hereditary pancreatitis; ref. 10). Unfortunately, even total pancreatectomy fails to ameliorate pain in some patients, in whom pain pathways have already undergone central sensitization (11).

\section{Chemical pancreatectomy}

Realizing that the autodigestive component of pancreatitis emanates from pancreatic acinar cells, Saleh et al. (12), in this issue of the JCI, report on their reasoning that ablation of the acinar cells may eliminate the initiating event in pancreatitis. Pancreatic acinar cells are generally 


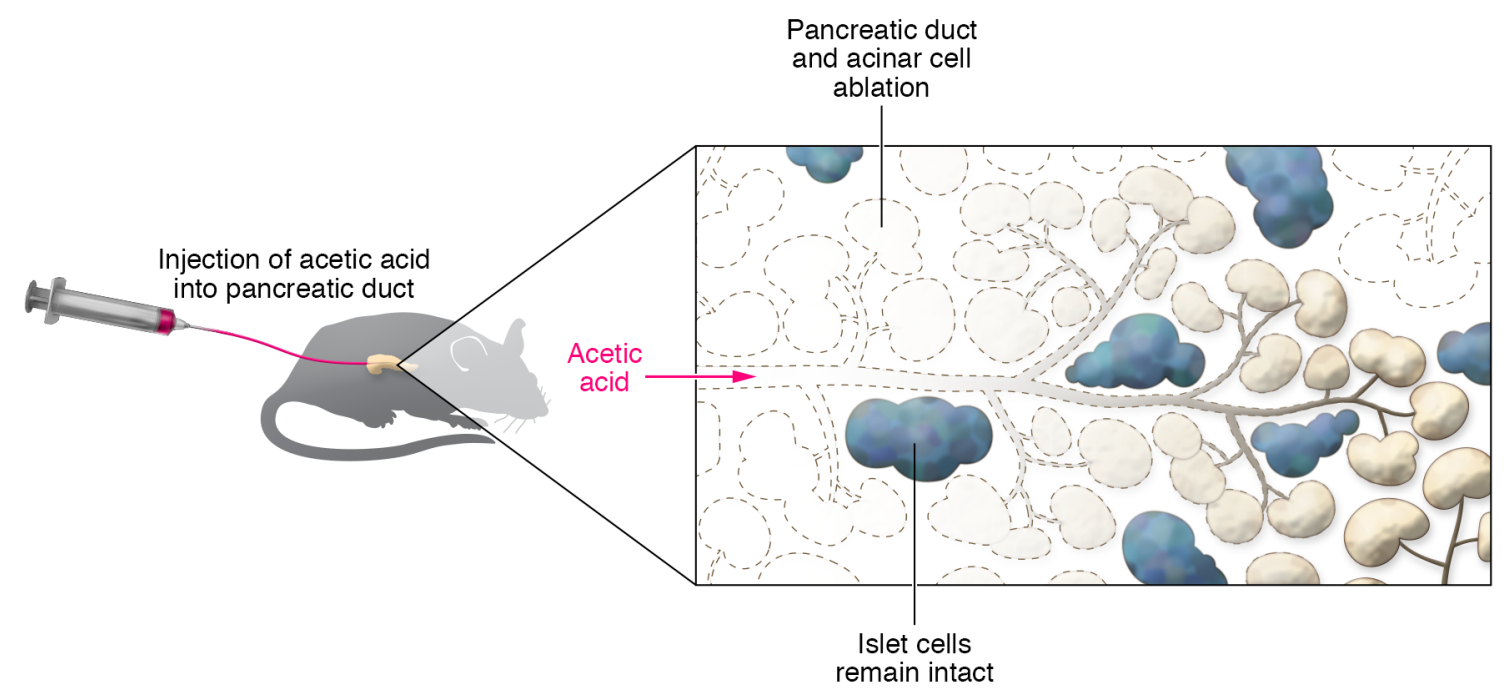

Figure 1. Pancreatic duct injection of acetic acid destroys the exocrine pancreas, but spares the islets. Saleh and colleagues injected saline or acetic acid $(0.2 \%)$ into the pancreatic duct of mice. The pancreatic histology appeared normal in saline-injected pancreas. In contrast, six months following acetic acid injection, the acinar and duct cells appeared absent, while the islets looked healthy (12).

believed to be the target of injury in pancreatitis. If the acinar cell and its accompanying minions (digestive enzymes) were removed, the pancreatic environment would become less hostile and subsequent injury could not incite a cascade of destructive autodigestive events.

As an alternative to surgical pancreatectomy, Saleh and authors chose to access the pancreas via pancreatic duct injection. They found that dilute acetic acid was lethal enough to kill cells that apposed the pancreatic duct (pancreatic acinar cells and duct cells themselves), but not so caustic that it destroyed more distant cells within the gland (i.e., islets; Figure 1). Within days of acetic acid injection, duct and acinar cells disappeared, while pancreatic islets remained intact. These changes were durable for at least six months. Importantly, the authors demonstrated that acetic acid injection effectively ablated the exocrine pancreas in both normal mice and mice with caerulein-induced chronic pancreatitis and ameliorated painlike behavior. They found that not only were pancreatic islets preserved, but animals secreted insulin and exhibited normal glucose tolerance. Thus, it appeared that acetic acid ablation did not adversely affect pancreatic endocrine function. Similarly to what occurred in mice, acetic acid injection was also shown to ablate pancreatic ducts and acinar cells, yet spare the islets, in a nonhuman primate model using
Cynomolgus macaques. The authors refer to this technique as "chemical pancreatectomy" and propose that acetic acid injection may be accomplished in humans via endoscopic pancreatic duct injection as in endoscopic retrograde cholangiopancreatography (ERCP) (12).

At first glance, intentionally destroying the entire exocrine pancreas seems like a bad thing to do. After all, the pancreas serves a useful purpose and, in the absence of pancreatic exocrine secretion, malabsorption of food and essential nutrients leads to malnutrition and vitamin deficiencies and, if untreated, may even be fatal. Although pancreatic enzyme replacement therapy ameliorates most of these effects, rarely does it normalize malabsorption (13). Nevertheless, many patients with chronic pancreatitis have exocrine insufficiency at the time of diagnosis, so eliminating any remaining pancreatic function when they already require lifetime pancreatic enzyme therapy may not be a prohibitive problem.

\section{Important physiological observations}

This study provides several physiological insights. First, it appears that pancreatic duct and acinar cells can be permanently destroyed by a single caustic injection. All exocrine cells are open to the duct lumen, where they could come into direct contact with acetic acid. It is now apparent that the regenerative capacity of the pancreas is eliminated when duct and acinar cells are destroyed. Following high doses of the cholecystokinin analogue, caerulein, which kills only acinar cells (14), the pancreas completely regenerated within a week, indicating that acinar cells repopulate, possibly from some dedifferentiated acinar cells that resemble duct cells (15-17). It appears that acetic acid instillation destroyed any remaining potentially regenerative cells, since neither acinar nor duct cells were seen after six months. Interestingly, injection of citric acid at similar $\mathrm{pH}$ did not exert the same effect, so the mechanism by which acetic acid ablates duct and acinar cells is unknown (12).

A second important physiological observation is that islets seem to do fine without surrounding acinar cells (12). Although, more detailed studies of endocrine function are warranted, the normal appearance and robust insulin secretion suggest that islets are not adversely affected by the destruction of exocrine cells. Retaining islet function in the setting of chronic pancreatitis is a notable achievement, since during the natural progression of human chronic pancreatitis, islets are damaged by ongoing inflammation. Halting islet (particularly $\beta$ cell) loss should stop, or at least delay, the onset of diabetes mellitus.

Remarkably, in their models of chronic pancreatitis, pain also appeared to be reduced following chemical pancreatec- 
tomy (12). Although the mechanism by which pain reduction occurs is unknown, the authors demonstrated both improvement in provoked pain responses and reduced mRNA levels of the neuroinflammatory mediator, calcitonin gene-related peptide (CGRP), in sensory dorsal root ganglia, suggesting that the pain resulted from ongoing pancreatic inflammation. However, achieving similar results in human chronic pancreatitis faces some major hurdles. Most patients with chronic pancreatitis have longstanding disease with difficult to treat pain. Since complete surgical removal of the pancreas fails to relieve pain in some patients (11), it is likely that chemical pancreatectomy would fall short in providing relief to those individuals also. Perhaps it will be possible in the future to predict those who might respond.

\section{An unconventional approach that just might work}

To determine whether this approach may translate to patients, clinicians will also have to overcome a number of technical challenges regarding the delivery of acetic acid. Upon direct exposure, acetic acid caused acinar cell necrosis and inflammation that lasted for at least four weeks (12). There is no reason to expect that iatrogenic pancreatitis would not also occur in humans. Even in a controlled, clinical settings, how physicians might manage pancreatitis induced by acetic acid treatment remains to be determined. Finally, in some patients, the entire pancreatic duct can be difficult to access endoscopically due to altered anatomy, such as strictures, pancreatic stones, and duct discontinuity. Leaving any acinar cells behind would result in an incomplete pancreatectomy and could limit the effectiveness of the procedure. Even in the pristine conditions of experimentally induced pancreatitis in mice and macaques, it was unclear whether all the acinar cells were destroyed. Despite these reservations, Saleh et al. (12) present a unique approach to a debilitating disease for which we have very limited therapeutic options. Should follow-up studies support these early preclinical findings, in selected patients, such as those with early, or who are at risk for, chronic pancreatitis, chemical pancreatectomy is conceivable.

Now back to our story. The doctor replies, "The injection will destroy his pancreas, so he won't have more attacks of pancreatitis and he won't develop diabetes." And it just might work.

\section{Acknowledgments}

This work was supported by NIH grants R01 DK 120555, 125308, and 124474 and the Department of Veterans Affairs.

Address correspondence to: Rodger A. Liddle, Box 103859, 1033A Genome Science Research Building - 1, 905 LaSalle Street, Duke University Medical Center, Durham, North Carolina 27710, USA. Phone: 919.681.6380; Email: rodger.liddle@ duke.edu.

1. Beyer G, et al. Chronic pancreatitis. Lancet 2020;396(10249):499-512.

2. Whitcomb DC, et al. Hereditary pancreatitis is caused by a mutation in the cationic trypsinogen gene. Nat Genet. 1996;14(2):141-145.

3. Witt $\mathrm{H}$, et al. Mutations in the gene encoding the serine protease inhibitor, Kazal type 1 are associated with chronic pancreatitis. Nat Genet. 2000;25(2):213-216.

4. Masson E, et al. Association of rare chymotrypsinogen C (CTRC) gene variations in patients with idiopathic chronic pancreatitis. Hum Genet.
2008;123(1):83-91.

5. Rosendahl J, et al. Chymotrypsin C (CTRC) variants that diminish activity or secretion are associated with chronic pancreatitis. Nat Genet 2008;40(1):78-82.

6. Cohn JA, et al. Relation between mutations of the cystic fibrosis gene and idiopathic pancreatitis. N Engl J Med. 1998;339(10):653-658.

7. Sharer $\mathrm{N}$, et al. Mutations of the cystic fibrosis gene in patients with chronic pancreatitis. N Engl JMed.1998;339(10):645-652.

8. Lowenfels $\mathrm{AB}$, et al. Hereditary pancreatitis and the risk of pancreatic cancer. International Hereditary Pancreatitis Study Group. J Natl Cancer Inst. 1997;89(6):442-446.

9. de la Iglesia-Garcia D, et al. Efficacy of pancreatic enzyme replacement therapy in chronic pancreatitis: systematic review and meta-analysis. Gut. 2017;66(8):1354-1355.

10. Abu-El-Haija M, et al. The role of total pancreatectomy with islet autotransplantation in the treatment of chronic pancreatitis: A report from the International Consensus Guidelines in chronic pancreatitis. Pancreatology. 2020;20(4):762-771.

11. Bouwense SA, et al. Altered central pain processing after pancreatic surgery for chronic pancreatitis. Br J Surg. 2013;100(13):1797-1804.

12. Saleh M, et al. Chemical pancreatectomy treats chronic pancreatitis while preserving endocrine function in preclinical models. JClin Invest. 2021;131(3):e43301.

13. Forsmark CE. Diagnosis and management of exocrine pancreatic insufficiency. Curr Treat Options Gastroenterol. 2018;16(3):306-315.

14. Reid LE, Walker NI. Acinar cell apoptosis and the origin of tubular complexes in caeruleininduced pancreatitis. Int J Exp Pathol. 1999;80(4):205-215.

15. Strobel O, et al. In vivo lineage tracing defines the role of acinar-to-ductal transdifferentiation in inflammatory ductal metaplasia. Gastroenterology. 2007;133(6):1999-2009.

16. Jensen JN, et al. Recapitulation of elements of embryonic development in adult mouse pancreatic regeneration. Gastroenterology. 2005;128(3):728-741.

17. Fendrich $\mathrm{V}$, et al. Hedgehog signaling is required for effective regeneration of exocrine pancreas. Gastroenterology. 2008;135(2):621-631. 